\title{
X-ray diffractometry of small defects in layered systems
}

\author{
V Holýt, J Kubĕnat, E Abramof \$, A Pesek‡ and E \\ Koppensteiner\| \\ † Department of Solid State Physics, Faculty of Science, The Masaryk University, \\ Kotlárská 2, CS-611 37 Brno, Czechosiovakia \\ ¥ Institute of Experimental Physics, Johannes Kepler University, Altenberger Str. \\ 69, A-4040 Linz, Austria \\ II Institute of Semiconductor Physics, Johannes Kepler University, Altenberger Str. \\ 69, A-4040 Linz, Austria
}

\begin{abstract}
X-ray diffraction in thin layers and layered systems is described using the optical coherence approach and the semi-kinematical diffraction theory. Two defect models in thin layers are considered-the mosaic structure model and the model of interface roughness. For both defect models the reflection curves of a thin layer and a superlattice have been calculated and compared with double-crystal $x$-ray diffractometry results on superlattices and epitaxial layers. The distribution of the diffusely scattered intensity near a reciprocal lattice point has been calculated theoretically for both models and it has been proved experimentally by double- and triple-crystal diffractometry of epitaxial layers with mosaic structure. It has been demonstrated that the theory yields a tool for estimating the predominant defect type in a layered structure.
\end{abstract}

\section{Introduction}

X-ray diffractometry can be used not only for determining basic structural parameters of epitaxial layers (ELS) and superlattices (SLS) such as thicknesses and lattice parameters, but also for studying their defect structure. $\mathrm{X}$-ray diffraction in a thin layer can be described by means of the semi-kinematical approximation [1]. This method enables us to calculate the wavefield on the exit surface diffracted by a thin single crystal or layered system with structural defects, if the whole crystal thickness is much smaller than the $x$-ray extinction length. If the defects are randomly placed in the crystalline matrix a diffuse $x$-ray scattering (DXS) occurs. This phenomenon can be described within the semi-kinematical approximation using the optical coherence formalism [2]. The DXS study provides valuable information concerning the defect structure and symmetry.

In this paper two types of structural defects are considered:

(i) volume defects in ELS and SLS described by a mosaic defect model and

(ii) interface defects in SLS (roughness).

The mosaic structure of ELS and SLs has been studied in several papers (e.g. $[3,4]$ ) by means of double crystal (DCD) and triple crystal $x$-ray diffractometry (TCD). On

§ Permanent address: Instituto de Pesquisas Espaciais, CP515, 12201-S. J. Campos, SP, Brazil. the basis of DXS measurement by TCD the misorientation and sizes of the mosaic blocks can be estimated.

The influence of rough interfaces in an SL on X-ray diffraction has been studied theoretically calculating the $\mathrm{x}$-ray reflection curves considering random deviations of layer thicknesses [5-7]. In our previous papers $[8,9]$ these defects have been described using the coherence formalism and DXs from these defects has been investigated.

The aim of this paper is to show the possibility of using $D C D$ and $T C D$ for distinguishing particular defect types and to calculate the $\mathrm{x}$-ray reflection curves and DXS on the basis of both defect models. The theoretical calculations are compared with experimental results.

\section{Theoretical description}

In the semi-kinematical approach the amplitude of the wave diffracted from a crystalline layer in a certain point $r$ at the surface is given by the wavefield in the crystal along the line $l(\boldsymbol{r})$ going through $\boldsymbol{r}$ in the direction of the diffracted wave:

$$
D(\boldsymbol{r})=\pi K \chi_{h} / \gamma_{h} \int_{l(\boldsymbol{r})} \mathrm{d} \boldsymbol{r}^{\prime} D_{0}\left(\boldsymbol{r}^{\prime}\right) \exp \left[-\mathrm{i} \boldsymbol{i} \cdot \boldsymbol{u}\left(\boldsymbol{r}^{\prime}\right)\right]
$$

where $\chi_{h}$ is the appropriate Fourier component of the polarizability of the perfect crystal, $K=1 / \lambda$ is the vacuum wavevector length and $\gamma_{h}$ is the direction $c 0$ sine of the diffracted wave with respect to the inward 
surface normal. $D_{0}\left(r^{\prime}\right)$ is the transmitted wave in the crystal approximated by the incident wave with an extra absorption term. The displacement of the atoms from their ideal positions due to defects is described by the vector $\boldsymbol{u}\left(\boldsymbol{r}^{\prime}\right)$. We assume that $\boldsymbol{u}$ is a random vector and we restrict ourselves to the case that all the measured quantities are averaged over all macroscopically equivalent defect configurations. Thus, we assume the mean distance between two neighbouring defects much smaller than the irradiated sample volume. The statistical ensemble averaging will be denoted by \langle\rangle .

We describe the emitted $x$-ray wavefield by the following mutual coherence function [10] (MCF)

$$
\Gamma\left(\boldsymbol{r}, \boldsymbol{r}^{\prime}\right)=\left\langle D(\boldsymbol{r}) D\left(\boldsymbol{r}^{\prime}\right)^{\star}\right\rangle .
$$

Using (1) the MCF can be expressed as

$$
\begin{aligned}
& \Gamma\left(\boldsymbol{r}, \boldsymbol{r}^{\prime}\right)=\left(\pi K / \gamma_{h}\right)^{2}\left|\chi_{h}\right|^{2} \\
& \quad \times \int_{l(\boldsymbol{r})} \mathrm{d} \boldsymbol{x} \int_{l\left(\boldsymbol{r}^{\prime}\right)} \mathrm{d} \boldsymbol{x}^{\prime} \Gamma_{0}\left(\boldsymbol{x}, \boldsymbol{x}^{\prime}\right) C\left(\boldsymbol{x}, \boldsymbol{x}^{\prime}\right)
\end{aligned}
$$

where

$$
C\left(\boldsymbol{x}, \boldsymbol{x}^{\prime}\right)=\left\langle\exp \left[-\mathrm{i} \boldsymbol{h} \cdot\left(\boldsymbol{u}(\boldsymbol{x})-\boldsymbol{u}\left(\boldsymbol{x}^{\prime}\right)\right)\right]\right\rangle
$$

is the correlation function of the crystal deformation field and $\Gamma_{0}\left(x, x^{\prime}\right)$ is the MCF of the incident beam.

The coherence approach is suitable for calculating both the diffracted intensity and its angular distribution (DXs intensity). The intensity is simply given by $I(r)=$ $\Gamma(r, r)$ and its DXs intensity is [10]

$J(q)=\int_{S} \mathrm{~d}\left(x-x^{\prime}\right) \mathrm{d}\left(y-y^{\prime}\right) \Gamma\left(r-r^{\prime}\right) \exp \left[\mathrm{iq} \cdot\left(\boldsymbol{r}-\boldsymbol{r}^{\prime}\right)\right]$

provided that the diffracted beam is homogeneous. The integration region $S$ is the exit crystal surface. Then, according to $[2,10]$, the DXS intensity measured by a TCD diffractometer is given by an integral of $J(q)$ over $q_{y}$ where the $y$ axis is perpendicular to the plane of diffraction. The resulting quantity is a function of two independent variables-angle of incidence of the primary beam $\alpha$ and the remaining coordinate $q_{x}$ of $q$. This quantity represents a distribution of the diffusely scattered intensity near the reciprocal lattice point in the plane of diffraction. The appropriate transformation formulae from $\alpha$ and $q_{x}$ to coordinates $q_{1}$ and $q_{2}$ in the reciprocal space can be found in [2].

The formalism presented here can be used not only for description of $\mathrm{x}$-ray diffraction in single heteroepitaxial layers but also for periodical superlattices. It has been shown in [9] that in the two-wave approximation (i.e. not far from the satellite diffraction maximum) a periodical SL can be treated as a homogeneous crystal, whose Fourier coefficient $\chi_{h}$ is replaced by the coefficient $\chi_{h H}$ from the following expression for the susceptibility $\chi(\boldsymbol{r})$

$$
\chi(\boldsymbol{r})=\sum_{h H} \chi_{h H} \exp [-\mathrm{i}(\boldsymbol{h} \cdot \boldsymbol{r}+H z)] .
$$

This series accounts for both the 'rapid' periodicity of the mean SL crystal lattice and the 'slow' artificial SL periodicity. The three-dimensional reciprocal space created by vectors $h$ is constructed on the basis of the average crystal lattice with lattice parameter $d=\left(T_{\mathrm{A}} d_{\mathrm{A}}+T_{\mathrm{B}} d_{\mathrm{B}}\right) /\left(T_{\mathrm{A}}+T_{\mathrm{B}}\right)$, where $T_{\mathrm{A}, \mathrm{B}}$ and $d_{\mathrm{A}, \mathrm{B}}$ are the thicknesses and lattice parameters of both phases $A, B$ building the SL (a lateral relaxation is not considered). The values $H=m /\left(T_{\mathrm{A}}+T_{\mathrm{B}}\right), m$ is an arbitrary integer, represent a one-dimensional reciprocal space in the growth direction ( $z$ axis).

Considering (4) the above formalism can also be applied for a periodical SL, in the formulae (1), (2) $\chi_{h}$ should be replaced by $\chi_{h H}$ and the correlation function $C\left(\boldsymbol{r}, \boldsymbol{r}^{\prime}\right)$ by

$$
\begin{gathered}
C_{S L}\left(\boldsymbol{r}, \boldsymbol{r}^{\prime}\right)=\left\langle\exp \left[-\mathrm{i} \boldsymbol{\mathrm { i }} \cdot\left(\boldsymbol{u}(\boldsymbol{r})-\boldsymbol{u}\left(\boldsymbol{r}^{\prime}\right)\right)\right]\right. \\
\left.\quad \times \exp \left[-\mathrm{i} H\left(t(\boldsymbol{r})-t\left(\boldsymbol{r}^{\prime}\right)\right)\right]\right\rangle .
\end{gathered}
$$

The random function $t(r)$ describes a discrepancy from the artificial SL periodicity, i.e. the interface roughness.

\section{The defect models}

In model $\mathrm{l}$, the crystal volume is divided into randomly misoriented mosaic blocks. For the sake of simplicity we assume that the random rotation vector $\varphi$ has the normal isotropic distribution with zero mean and mean square dispersion $\Delta^{2}$. Further, we neglect a possible anisotropy of the shape of the blocks, i.e. the probability of finding two arbitrary points $r$ and $r^{\prime}$ in the same block depends only on $\left|\boldsymbol{r}-\boldsymbol{r}^{\prime}\right|$. The mean block radius is denoted by $R$. The explicit formula for $C\left(\boldsymbol{r}, \boldsymbol{r}^{\prime}\right)$ is rather complicated; it will be published elsewhere [11]. In the case of an SL, with mosaic structure (a strained-layer SL) the influence of a possible interfacial roughness is very weak compared with that of the mosaic structure, therefore, $t(r)=0$ can be assumed. Using the theory above we obtain direct formulae for the diffracted intensity $I$ and its angular distribution $J$ as functions of $R$ and $\Delta$. Numerical simulations not shown here confirmed that the mosaic structure affects all the diffraction satellites of a SL in the same way, i.e. the FWHMS and shapes of all the satellites are identical.

The details of model II have been published in [9]. We assumed $u=0$, i.e. the 'rapid' periodicity is not disturbed and, therefore, the layers have an ideal crystal structure. The random profile $t\left(x, y, z_{j}\right)$ of the $j$ th interface in a SL has been characterized by the correlation function (5) containing two statistical parameters: the mean square roughness $\sigma_{1}^{2}$ of the first interface (near the substrate) and the lateral correlation length $\Lambda$. In the model we assumed that $\sigma_{j}^{2}=j \sigma_{1}^{2}$, i.e. the interface roughness grows towards the surface. The lateral roughness correlations are substantial for calculating the DXs intensity. In our model we assumed that the rough interface is described by a Poisson-like random process, thus, the lateral correlation in the same interface is $K\left(\left|\boldsymbol{r}-\boldsymbol{r}^{\prime}\right|\right)=\exp \left(-\left|\boldsymbol{r}-\boldsymbol{r}^{\prime}\right| / \Lambda\right)$. From the 


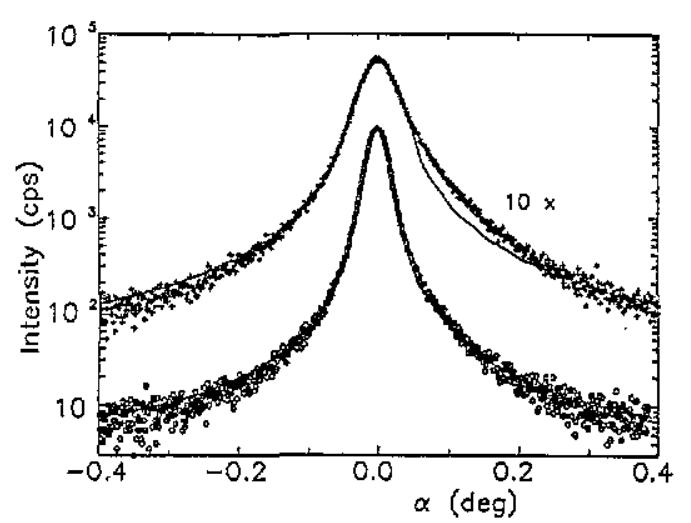

Figure 1. Double crystal reflection curves (CuKar radiation, the symmetrical 004 Bragg case of diffraction) measured on a ZnTe EL grown on a GaAs substrate. Only the layer diffraction peak is shown. Circles: before thinning, the layer thickness is $T=6.22 \mu \mathrm{m}$; crosses: after thinning, $T=3.50 \mu \mathrm{m}$. The full curves represent the fit of the theoretical curves. $\alpha$ is the angular deviation of the primary beam from the diffraction position in the layer.

theory it follows that within this model the influence of the defects grows with the diffraction satellite order $m$ approximately as $m^{2}$, i.e. the FWHMS of the satellites grow with increasing $m$. Therefore, if $m=0$, i.e. in the case of the main diffraction maximum, the roughness has no influence on the diffraction and the SL behaves as an ideal structure.

It is worth noting that for both models the integrated reflectivity of the diffraction maximum (or of the satellite for the case of a SL) is the same as that for an ideal structure. On the contrary, a possible interdiffusion taking place at interfaces in a SL diminishes the integrated reflectivity of the satellite.

\section{Examples}

We will show now the possibilities of the theory described above in analysing experimental $D C D$ and $T D C$ reflection curves.

The defect model I has been verified by DCD and TCD measurements of a $\mathrm{ZnTe}$ El grown by the hot-wall epitaxy technique on a GaAs substrate. Due to the great lattice misfit of $\mathrm{ZnTe}$ and $\mathrm{GaAs}$ (about $7.7 \%$ ) the $\mathrm{ZnTe}$ layer was fully relaxed and no strain gradient existed in the layer. Figure 1 shows the experimental DCD reflection curves of the layer before and after chemical thinning. The numerical fit of the theoretical curves with experimental ones yielded the following values:

for layer thickness $=6.22 \mu \mathrm{m}, R=(0.221 \pm 0.005)$ $\mu \mathrm{m}, \Delta=50^{\prime \prime} \pm 3^{\prime \prime}$

for layer thickness $=3.50 \mu \mathrm{m}, R=(0.104 \pm 0.005)$ $\mu \mathrm{m}, \Delta=65^{\prime \prime} \pm 6^{\prime \prime}$.

The experimental details will be published in a forthcoming paper [11].

Figure 2 shows the Dxs contour map measured by TC.D and the map calculated on the basis of the $R, \Delta$ values above. (a)

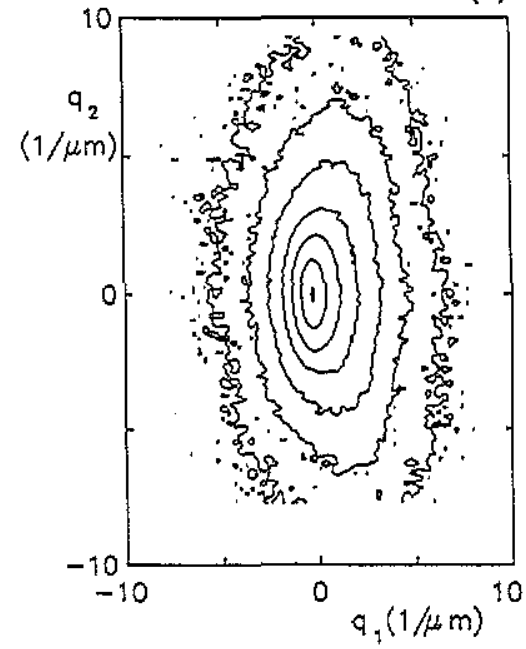

(b)

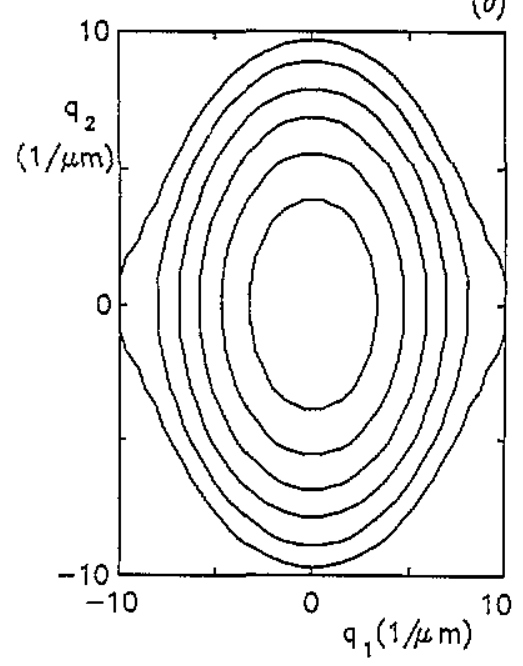

Figure 2. (a) The oxs intensity distribution near the reciprocal lattice point 004 of the layer according to figure 1 (layer thickness $3.50 \mu \mathrm{m}$ ) measured by TCD using CuK $\alpha_{1}$ radiation in the symmetrical Bragg case diffraction. The coordinates $q_{1,2}$ express the deviation from the reciprocal lattice point in directions parallel and perpendicular to the diffraction vector, respectively. The neighbouring contours represent the DXS intensity ratio $10^{1 / 2}$. (b) The same situation as in (a), the contours are calculated using the mosaic defect model a the values $R$ and $\Delta$ following from the DCD measurement.

The model of interface roughness has been proved by measurements of a SL AlAs/GaAs provided by courtesy of K Ploog (Stuttgart). The nominal thickness values for the GaAs and the AlAs layers are $T_{\mathrm{A}}=4.0 \mathrm{~nm}$ and $T_{\mathrm{B}}=5.4 \mathrm{~nm}$, and the whole stack contains 60 elementary $(A, B)$ cells. Figures 3 and 4 show its experimental $D C D$ reflection curves, the experimental details can be found in $[8,9]$. From both the satellites we obtained an estimate for roughness $\sigma_{1}=1.0 \pm 0.1$ monolayers. The determination of the correlation length $\Lambda$ was not successful as the statistical error resulting from the numerical least-square-fit procedure made about $60 \%$ of its value. The values of $\Lambda$ known from literature-about $100 \mathrm{~nm}$ or more-are very small and thus the use of a conventional $\mathrm{x}$-ray tube is not appropriate. 


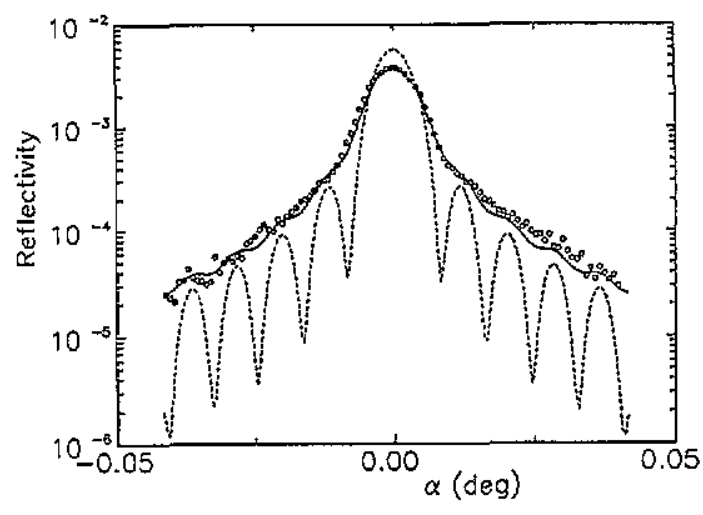

Figure 3. The DCD reflection curve of the first diffraction satellite measured in the symmetrical 002 Bragg case diffraction (CuK $\alpha_{1}$ radiation) on a periodical AlAs/GaAs superlattice (circles), the full curve is the best fit calculated from the theory, the broken curve represents the diffraction satellite of a perfect SL. For the sc parameters see text.

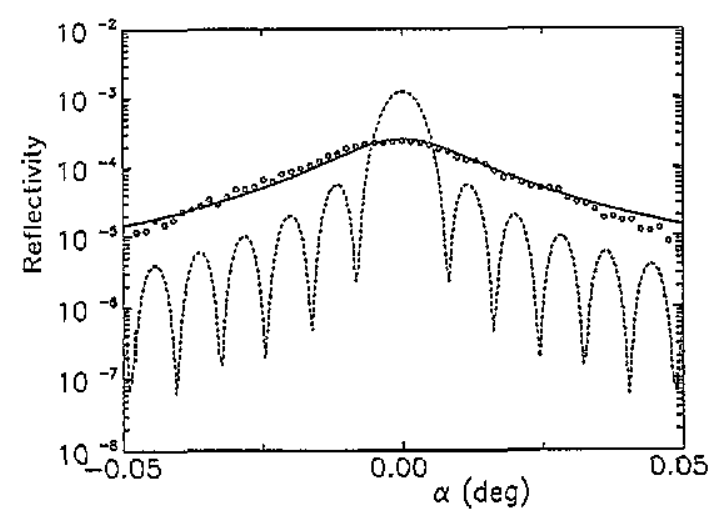

Figure 4. The same situation as in figure 3 but for the third satellite. It is obvious that the influence of the interface roughness on the satellite shape grows with the satellite order (compare with figure 3).

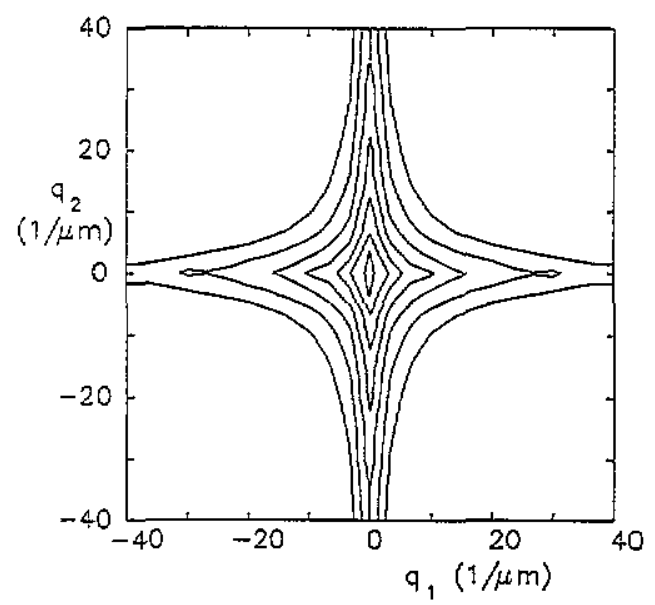

Figure 5. The DXs intensity distribution near the 002 reciprocal lattice point of the same st as in figures 3 and 4 calculated assuming rough interfaces with $\sigma=0.1$ monolayers and $\Lambda=0.1 \mu \mathrm{m}$. The neighbouring contours have the intensity ratio $10^{1 / 2}$.

In figure 5 the calculated equi-intensity contours are plotted for a DXs near a reciprocal lattice point due to rough interfaces in the same SL. From the theory it follows that the shape of the $J\left(q_{1}=0, q_{2}\right)$ dependence, i.e. the cut of the contour map along the $q_{2}$ axis yields a Fourier transformation of the lateral correlation function $K\left(\left|\boldsymbol{r}-\boldsymbol{r}^{\prime}\right|\right)$.

\section{Discussion}

In figure 1 it can be seen that the theory explains the shape of the DCD reflection curves of a mosaic EL quite well. Other measurements, which will be published elsewhere [11], have shown that the values $R$ and $\Delta$ obtained from those curves measured in various diffractions coincide within the error limits. The structure model of the mosaic blocks can be refined considering a possible strain deformation of the blocks and/or their plate-like shape.

The shapes of the measured (figure $2(a)$ ) and calculated (figure 2(b)) DXs intensity contour plots agree qualitatively. The parameters $R$ and $\Delta$ can be obtained by fitting the theoretical and experimental DXs plots with higher accuracy than from the DCD curves. This will be the subject of further investigations.

It is well known from the literature [12] that relaxed epitaxial layers grown on substrates with great lateral lattice misfit exhibit a significant structural inhomogeneity in the growth direction. It could be expected that towards the free surface of the layer its crystalline quality improves. This phenomenon was not considered in our structure model. The inhomogeneity can be studied by our method using various diffractions or wavelengths with various penetration depths.

The crystal structure of nearly strain-free sLs (such as AlAs/GaAs) is very good, so that the DXs caused by defects II could hardly be detected using a conventional $x$-ray source. The presence of these defects, however, can be stated by a DCD measurement with a usual $x$ ray tube. The diffracted intensity measured by a DCD is integrated over the Ewald sphere near the reciprocal lattice point (i.e. the x-ray detector with an open window sums all the waves propagating with various directions), so that this intensity is much more sensitive to a possible interface roughness than a DXs intensity measured by a TCD. Nevertheless, a direct measurement of the DXS by a TCD could bring direct information concerning the correlation properties of the roughness at the same interface.

The above theory could also be applied for $h=$ 0 . This case corresponds with the small-angle $x$-ray reflection occuring both in crystalline and in amorphous SLS. Since the theory does not include the total specular $\mathrm{x}$-ray reflection occurring at the sample surface, the results would be incorrect for incidence angles near the critical angle of the total $\mathrm{x}$-ray reflection.

\section{Conclusion}

The semi-kinematical description of $x$-ray diffraction in thin layers with defects and the optical coherence 
approach make it possible to calculate the diffracted intensity and its angular distribution (diffuse $x$-ray scattering) for various defect models. The theory predicts the shapes of diffraction maxima and their integrated intensities, so that the predominating defect type can be estimated. A detailed numerical analysis of $D C D$ reflection curves and DXS distribution in the reciprocal plane enables us to state mean values of the defect parameters, if an appropriate defect model is assumed. Therefore, for a complete analysis of the defects structure of a layered system a combination of various experimental methods is desirable. Application of the theory for real superlattices with nearly perfect layers ( $\mathrm{A}^{\mathrm{III}} \mathrm{B}^{\mathrm{V}} \mathrm{SLS}$ ), however, demands for a more powerful $\mathrm{X}$-ray source.

\section{References}

[1] Petrashen P V 1974 Fiz. Tverd. Tela 162168
[2] Holý V and Kuběna J 1992 Phys. Status Solidi

[3] Fewster P F 1989 J. Appl. Cystallogr. 2264

[4] Fewster P F 1991 J. Appl. Crystallogr. 24178

[5] Appel A, Bonse U and Staudenmann J I 1990 Z. Phys. B 81371

[6] Koshiba S, Nano S, Tsuda O, Watanabe Y, Sakurai Y, Sakaki H, Kawata H and Ando M 1989 J. Cryst. Growth 9551

[7] Fewster P F 1988 J. Appl. Crystallogr. 21524

[8] Holý V, Kubẽna J and Ploog K 1990 Phys. Status Solidi b 162347

[9] Holý V, Kubęna J and Ploog K 1992 Superlattices Microstructures at press

[10] Holý V and Gabrielyan K T 1987 Phys. Status Solidi b 14039

[11] Abramof E, Holý V, Koppensteiner E and Pesek A to be published

[12] Kudlek G, Presser N, Gutowski J, Hingeri K, Abramof E, Pesek A, Pauli H and Sitter H $1992 \mathrm{~J}$. Cryst. Growth 117290 